\title{
sms4science: Korpusdaten, Literaturüberblick und Forschungsfragen
}

\author{
Adrian Stähli, Christa Dürscheid \& Marie-José Béguelin (Bern/Zürich/Neuchâtel)
}

\begin{abstract}
This paper discusses some methodological issues related to current research on SMS communication in Switzerland, an ambitious research project situated in the context of the international project sms4science. Thus far, the analyses of the Swiss data focus on numerous inner-linguistic features, variational aspects and language contact phenomena; each of which is of the utmost interest for multilingual Switzerland (cf. the five articles contained in this issue). In the following pages we first provide some background information about the project and the constitution of the Swiss SMS-corpus. We then present some methodological demands and problems, which are discussed on the basis of a dialogic sub-corpus. In the final paragraphs of this introductory article, we outline the state of affairs in the field of the linguistic study of SMS communication and highlight some research questions that would be of significant interest for the further analysis of the Swiss corpus.
\end{abstract}

\section{Spenden Sie der Forschung Ihre SMS!}

Die Beiträge des vorliegenden Heftes situieren sich im Kontext des internationalen Forschungsprojekts sms4science, das vom Zentrum für maschinelle Sprachverarbeitung der Katholischen Universität Louvain (CENTAL) koordiniert wird (siehe unter www.sms4science.org). Die Anfänge dieses Projektes gehen zurück auf das Jahr 2004. Damals lancierte das CENTAL unter der Leitung von Cédrick Fairon und Louise-Amélie Cougnon die Kampagne "Faites don de vos SMS à la science !". Über die Medien luden sie alle HandynutzerInnen dazu ein, von jeder versandten SMS eine Kopie an eine Gratisnummer zu schicken und im Internet einen anonymen Fragebogen auszufüllen (vgl. hierzu den Beitrag von Louise-Amélie Cougnon und Thomas François in diesem Heft). Nach Abschluss der Sammelaktion lagen über 75'000 SMS vor, von denen ca. 30'000 in einem Korpus zusammengestellt wurden, das nun für wissenschaftliche Untersuchungen zur Verfügung steht. In einem zweiten Schritt nahm die Forschergruppe des CENTAL Kontakt mit Wissenschaftlern anderer Länder auf, lud diese zur Kooperation ein und stellte ihre Methodologie für weitere Sammelaktionen bereit. Mittlerweile sind zwölf Länder dem Projekt angeschlossen; die Palette der beteiligten Nationen reicht von La Réunion bis Grossbritannien, von Kanada bis Griechenland. Alle Kooperationspartner legen, wie in Belgien, den Schwerpunkt auf die Sammlung von solchen Textnachrichten, die über Mobilfunkgeräte (Handys) verschickt werden. Zwar ist dies keineswegs immer der Fall; bekanntlich werden SMS auch am Computer geschrieben, versendet und empfangen. Dennoch stehen im Projekt per Handy verschickte SMS im Zentrum, da hier spezifische, vom Computer distinkte mediale Bedingungen gelten, die wiederum $\mathrm{zu}$ einem spezifischen Sprachgebrauch führen können (s.u.). 
Auch die Schweiz trat dem Projekt sms4science bei: Im Jahr 2008 wurde ein Kooperationsvertrag mit dem CENTAL geschlossen und von Christa Dürscheid und Elisabeth Stark (Universität Zürich) sowie Marie-José Béguelin und Simona Pekarek Doehler (Universität Neuchâtel) unterzeichnet, im Herbst 2009 startete die SMS-Sammelaktion in der Schweiz: Die Bevölkerung wurde aufgerufen, eine SMS mit Angabe der E-Mail-Adresse an eine Gratisnummer der Swisscom zu schicken. Wer sich beteiligte, erhielt sofort eine automatisch erstellte Antwort-SMS mit weiteren Informationen und wenig später über E-Mail den Link zu einem Online-Fragebogen. Sobald der Fragebogen eingegangen war und die Teilnehmer fünf oder mehr SMS eingeschickt hatten, nahmen sie an einer Verlosung teil, bei der wöchentlich ein iPhone zu gewinnen war. Damit sollte erreicht werden, dass sich möglichst viele Personen an der Sammelaktion beteiligten. Selbstverständlich wurde den Teilnehmern absolute Anonymität zugesichert: Die Swisscom verpflichtete sich, die eingesandten SMS vertraulich zu behandeln und sie den Forschergruppen in Zürich und Neuchâtel zusammen mit den Fragebogen ohne Angabe der Handy-Nummer zur Verfügung zu stellen. Wir unsererseits garantierten den Beteiligten, dass alle Personendaten (z.B. Telefonnummer, E-Mail-Adresse), die Rückschlüsse auf die Verfasser zuliessen, getilgt würden. Zwischen November 2009 und Januar 2010 wurden auf diese Weise rund 27'000 SMS gesammelt. Weiter unten gehen wir auf die einzelnen Schritte in der Datensammlung und -anonymisierung genauer ein (siehe auch die Website des Projekts www.sms4science.ch); hier sei nur ein Beispiel für eine der vielen SMS gegeben, die in das Schweizer Korpus eingegangen sind. Dabei handelt es sich um eine SMS, die in Schweizerdeutsch verfasst wurde (ergänzt um englische Sequenzen):

(1) oh schnuggiputz hallo, i miss you! Du hesches bald gschafft :-) vernünftig bisch mitem zug gange, super! $\mathrm{Cu}$ soon :-*.

Nach den ersten Arbeiten an den erhaltenen Daten, aber auch, um langfristig ein online benutzbares Referenzkorpus schweizerischer SMS aufzubauen (vgl. hierzu Abschn. 2), fand im Anschluss an die SMS-Kampagne ein Workshop statt. Dieser wurde - mit Unterstützung der Faculté des Lettres et Sciences humaines - am 16. Juni 2010 zusammen mit den internationalen Kooperationspartnern in Neuchâtel durchgeführt. ${ }^{1}$ Er diente vor allem dazu, ein Treffen aller am Projekt beteiligten Personen zu ermöglichen, sich über die aktuelle Datenlage und erste Auswertungsresultate auszutauschen und die Planung eines gemeinsamen Forschungsprojektes in Angriff zu nehmen. ${ }^{2}$ Diskutiert wurden anlässlich dieses Workshops ethische Fragen, welche eine langfristige Publikation des Korpus betreffen, aber auch die Forschungsfragen, die (z.B. im Rahmen von Dissertationsprojekten) auf der Basis der Korpusdaten bearbeitet werden sollen. Dazu gehören u.a. die folgenden beiden Themenkreise: sprachliche Merkmale in Schweizer SMS (insbesondere auf grammatischer Ebene); Variation in Schweizer SMS (v.a. im Hinblick auf Mehrsprachigkeit und Code-Switching). Das vorliegende Heft stellt die ersten Resultate dieser Workshop-Gespräche vor und rückt dabei, wie bereits am Titel zu sehen, den Umstand ins Zentrum, dass die SMS-Forschung, über die hier berichtet wird, in einem mehrsprachigen Land durchgeführt wird und im Korpus Daten in allen vier Nationalsprachen vorliegen. Die Inhalte der einzelnen Beiträge werden in Abschn. 5 skizziert; zunächst seien einige Informationen zur Zusammenstellung und Aufbereitung des SMS-Korpus gegeben (Abschn. 2). Im Anschluss daran stellen wir ein Subkorpus vor, das den SMS-Dialog zweier Personen (nennen wir sie Max und Petra) illustriert (Abschn. 3). Ausserdem wird ein knapper Literaturüberblick zur SMS-Forschung gegeben, und es werden

\footnotetext{
1 Der Workshop eröffnete gleichzeitig die "Etats généraux du français en Francophonie", eine Reihe von insgesamt 14 akademischen Veranstaltungen, die anlässlich des 13. Gipfels der Francophonie in Montreux stattfanden.

2 Ein solches Projekt wurde im Juni 2011 vom Schweizerischen Nationalfonds bewilligt.
} 
sms4science: Korpusdaten, Literaturüberblick und Forschungsfragen

einige der Forschungsfragen benannt, die auf der Basis des Schweizer Korpus untersucht werden sollen (Abschn. 4).

\section{Korpuszusammenstellung und Korpusaufbereitung}

Nach Ablauf der Sammelaktion lagen uns, wie bereits erwähnt, knapp 27'000 SMS vor. Nach der ersten Sichtung der Daten zeigte sich aber schnell, dass es, wie auch in Belgien, nicht möglich war, alle diese SMS in das Korpus aufzunehmen. Die Gründe hierfür sind vielfältig: So waren aufgrund technischer Probleme einige SMS doppelt vorhanden. Andere waren nicht, wie vorgegeben, auf dem Handy, sondern per Computer geschrieben (erkennbar z.B. am Text sent by xtrazone). Sie kamen deshalb nicht für unsere Untersuchungszwecke in Frage. Weiter wurden Nachrichten an die Swisscom-Nummer geschickt, die von SMSComputern erstellt worden waren (z.B. automatische Benachrichtigungen über den Erhalt einer MMS). Auch diese Daten mussten aussortiert werden, so dass sich die Anzahl der SMS im Korpus letztendlich auf 23'988 reduzierte (mit einem Total von ca. 480'000 Wörtern). Die Verteilung der Sprachen in diesen SMS gestaltet sich wie folgt: Standarddeutsch: 4'734, Schweizerdeutsch: 13'434, Französisch: 4'505, Italienisch: 474, Rätoromanisch: 214, Englisch: 561, andere Sprachen: $66 .^{3}$

Die meisten Teilnehmer stellten dem Projekt ein bis fünf SMS zur Verfügung, über 80 Personen schickten aber auch mehr als 50 Nachrichten, und eine Person gar 413. Fast 50\% (1'311) beantworteten zusätzlich auch den Online-Fragebogen, machten also Angaben zu Geschlecht, Alter, Beruf, Ausbildung, Muttersprache, aber auch zu ihren SMSSchreibgewohnheiten und ihren Lesepräferenzen. Weil vor allem diejenigen Teilnehmer, die viele SMS einschickten, auch den Fragebogen ausfüllten, liegen uns zu ca. $75 \%$ der eingegangenen Nachrichten Personendaten vor. Aufgrund dieser Angaben wissen wir z.B., dass ca. 45\% der Teilnehmer zwischen 21 und 30 Jahre alt sind, aber auch, dass fünf Personen teilgenommen haben, die älter als 70 sind.

Im Folgenden wird eine erste Übersicht über die Daten präsentiert (vgl. hierzu ausführlich Dürscheid/Stark 2011). Dabei ist zu beachten, dass nicht alle Personen, die den Fragebogen ausfüllten, auch alle Fragen beantwortet haben. Deshalb entspricht das Total jeweils nicht der Summe der Einzelwerte.

\begin{tabular}{|l|c|c|c|c|c|c|}
\hline & SMS & Wörter & Teilnehmer & Fragebogen & \multicolumn{2}{|c|}{ Geschlecht } \\
\hline & & & & & $\mathrm{F}$ & $\mathrm{M}$ \\
\hline Total & $23^{\prime} 988$ & ca. 480'000 & $2^{\prime} 627$ & $1^{\prime} 311$ & 831 & 477 \\
\hline
\end{tabular}

Tabelle 1: Überblick Korpusdaten sms4science.ch

\begin{tabular}{|c|c|c|c|c|c|c|}
\hline $10-19$ & $20-29$ & $30-39$ & $40-49$ & $50-59$ & $60-69$ & $70-$ \\
\hline 245 & 599 & 190 & 152 & 80 & 38 & 5 \\
\hline
\end{tabular}

Tabelle 2: Alter der Teilnehmer (sofern die Frage beantwortet wurde)

\begin{tabular}{|l|c|c|c|c|c|c|}
\hline & $\begin{array}{c}\text { Schweizer- } \\
\text { deutsch }\end{array}$ & Standarddeutsch & $\begin{array}{c}\text { Franzö- } \\
\text { sisch }\end{array}$ & Italienisch & $\begin{array}{c}\text { Rätoroma- } \\
\text { nisch }\end{array}$ & Andere \\
\hline Total & 889 & 161 & 256 & 54 & 26 & 125 \\
\hline
\end{tabular}

Tabelle 3: Muttersprache (mehrere Angaben möglich)

\footnotetext{
3 Die Zahlen basieren auf der automatisierten Spracherkennung (siehe dazu weiter unten). Vorweg wurden viele SMS aber auch schon manuell durchgesehen. In diesem Rahmen wurden 66 SMS identifiziert, die in anderen als den hier genannten Sprachen verfasst sind. Vermutlich liegt die Zahl aber wesentlich höher.
} 
Wie die Zahlen zeigen, gaben die meisten Teilnehmer an, dass Schweizerdeutsch (genauer: ein Deutschschweizer Dialekt) ihre Muttersprache sei. Das zeigt sich auch im Korpus: Rund drei Viertel der deutschsprachigen SMS sind in Schweizerdeutsch verfasst. Dies ist - wie auch in der Forschung immer wieder betont wird (vgl. Siebenhaar 2006) - ein Beleg dafür, dass sich die mediale Diglossie in der Deutschschweiz (d.h. gesprochen wird Dialekt, geschrieben wird Standarddeutsch) heute anders gestaltet, als dies noch vor Jahren der Fall war. Pointiert gesagt: Gesprochen wird Dialekt, geschrieben wird - zumindest in der privaten Kommunikation unter Jugendlichen - auch Dialekt. ${ }^{4}$

Damit kommen wir zur Datenanonymisierung, die gerade in einem solchen Projekt, das mit hochsensiblen Daten arbeitet, von immenser Wichtigkeit ist. Alle Zahlen im Korpus, die mehr als zwei Ziffern enthielten (z.B. Telefonnummern und Bankkontodaten), mussten durch Variablen ersetzt werden, auch die Angaben zu Strassen, E-Mail-Adressen und Nachnamen wurden anonymisiert. Die Vornamen dagegen wurden nur rotiert. Aus François wurde also Peter (fiktives Beispiel), aus Monique wurde Petra. Auf diese Weise war es möglich, die Dialogizität der SMS zu rekonstruieren, während gleichzeitig die Anonymität der Schreiber gewahrt bleibt. Ein weiterer Arbeitsschritt stellte die automatische Spracherkennung dar. Hierzu verwendeten wir Referenzlisten, anhand derer der Computer erkennen kann, welche Buchstabenkombinationen für eine Sprache typisch sind (sogenannte N-Gramme). Die auf der Basis dieser Referenzlisten durchgeführte Spracherkennung gestattete eine erste Sichtung der Daten, die Ergebnisse müssen nun aber noch manuell nachbearbeitet werden. So treten in vielen SMS Code-Switching-Phänomene auf (vgl. Sounds good;-) freu mich!!), was im Einzelfall die Zuordnung zu einer Sprache erschwert. ${ }^{5}$ Ein weiteres Problem stellt sich, wenn in einer SMS Lehnwörter verwendet werden (z.B. merci). Gerade wenn die Gesamtwortzahl in einer solchen SMS gering ist, ist es keineswegs eindeutig, welches hier die Hauptsprache ist.

Inzwischen sind die Korpusarbeiten schon weit fortgeschritten, und das Korpus ist passwortgeschützt über jeden gängigen Browser im Internet aufrufbar. Als Arbeitsinstrument hierfür ist der Corpus Navigator im Einsatz, eine Benutzeroberfläche, welche Hans-Martin Lehmann (Englisches Seminar der Universität Zürich) entwickelte und die seinem Copyright unterliegt. Ein Vorteil dieser Art der Datenaufbereitung im Internet ist - im Gegensatz zu einer Datenbank auf CD-Rom - die Unabhängigkeit vom Betriebssystem des jeweiligen Benutzers. Ein weiterer Vorteil besteht darin, dass man technische Anpassungen nur auf dem Server ausführen muss, nicht aber beim einzelnen Nutzer.

Im Corpus Navigator sind jetzt schon einfache Suchabfragen möglich. So kann man die Frequenz von Zeichensequenzen überprüfen, von denen gemeinhin angenommen wird, es seien typische Schreibweisen der Handykommunikation, oder man kann alle konjugierten Formen eines Verbs ermitteln und auf diese Weise feststellen, wie häufig und in welchen Konstellationen ein bestimmtes Verb (z.B. lieben) im Korpus vorkommt. Um aber komplexere Suchabfragen durchführen zu können, muss das Korpus noch annotiert, d.h. auf grammatischer Ebene mit Metadaten versehen werden. Hierfür müssen zunächst alle SMS sprachlich und orthographisch in die Standardsprache übertragen (= "standardisiert") werden. Nur dann ist es möglich, die in den SMS vorkommenden Wortarten automatisch zu kennzeichnen ("part-of-speech-tagging"). Ein Satz, der in Schweizerdeutsch verfasst ist (z.B. Häsch ems gseit?), muss also zunächst standardisiert werden, denn nur in der Variante Hast

\footnotetext{
${ }^{4} \mathrm{Zu}$ diesem Ergebnis gelangt auch eine von Christa Dürscheid geleitete Nationalfondsstudie. Im Rahmen dieses Projekts wurden die Freizeittexte (z.B. E-Mails, SMS, Chat-Beiträge) von 347 Schülern aus den Kantonen Zürich und Zug ausgewertet und mit Schultexten (z.B. Deutschaufsätzen) verglichen (vgl. Dürscheid/Brommer/Wagner 2010).

5 Von Code-Switching sprechen wir nur dann, wenn fremdsprachliche Elemente vorkommen, die nicht als Lehnwörter in den Wörterbüchern der Ausgangssprache erfasst sind, wenn also Ad-hoc-Entlehnungen auftreten.
} 
Du es ihm gesagt kann der Tagger die grammatische Person identifizieren (Beispiel von Simone Ueberwasser, p.c.). Auf diese Weise wird es auch möglich sein, trotz grosser dialektaler Unterschiede jeweils das finite Verb im Satz zu erkennen - und das wiederum ist eine Voraussetzung dafür, dass quantitative Aussagen zur Verbstellung möglich sind, aber auch z.B. zu der Frage, wie häufig finite Verben überhaupt vorkommen.

Doch nicht nur quantitative Auswertungen werden im Korpus sms4science zur Anwendung kommen, auch qualitative Untersuchungen sollen durchgeführt werden. Hierfür eignen sich die drei Subkorpora, die uns von Einzelpersonen zur Verfügung gestellt wurden und in einer separaten Datenbank erfasst sind, in hervorragender Weise. Anhand dieser Daten lässt sich z.B. untersuchen, wie die Beziehung zwischen den Interaktionspartnern ausgehandelt wird, welche kommunikativen Unterschiede im Vergleich zu Face-to-Face-Gesprächen auftreten und welchen Einfluss es auf den Sprachgebrauch hat, wenn die SMS in Sekundenschnelle hin und her wechseln. Ein Beispiel für ein solches Subkorpus wird im Folgenden kurz vorgestellt.

\section{Das Subkorpus Max und Petra}

Im Anschluss an die von uns im Kontext von sms4science.ch lancierte Pressekampagne wurde Marie-José Béguelin am 21. November 2009 von einem Schweizer kontaktiert, der dem Projekt ausserhalb der automatisierten Datensammlung ein Korpus von dialogischen SMS-Sequenzen zwischen ihm und seiner Partnerin zur Verfügung stellen wollte. Da solch dialogische Daten ein Desiderat für die Erforschung der SMS-Kommunikation darstellen, nahmen wir dieses Angebot gerne an und erhielten bereits am Folgetag ein SMS-Korpus im Word-Format, bestehend aus über 250 Nachrichten. Alle SMS sind in französischer Sprache abgefasst, in relativ konventioneller Graphie, die nur selten von den für SMS typischen Schreibweisen (s.u.) Gebrauch macht. Die Korrespondenz der zur Verfügung gestellten Kurznachrichten erstreckt sich über einen Zeitraum von knapp fünf Monaten (vom 26. Juni bis zum 21. November 2006). ${ }^{6}$ Den Informationen zufolge, die der 'Spender' uns mit dem Korpus zukommen liess, handelt es sich bei den Korrespondenten um einen ca. 60 Jahre alten Mann (den Absender selbst), von deutscher Muttersprache, und eine Frau um die 50 Jahre mit französischer Muttersprache. Beide werden im Folgenden mit den Pseudonymen Max und Petra benannt. Der Beginn der Korrespondenz geht, soweit sich dies aufgrund der Texte beurteilen lässt, mit dem Beginn einer Liebesbeziehung zwischen den beiden Korrespondenten einher.

Bevor wir im Folgenden einige Auszüge daraus präsentieren, sei noch ein Wort zur Authentizität der Nachrichten gesagt: Zwar können wir davon ausgehen, dass es sich jeweils um authentische Nachrichten handelt, allerdings wissen wir nicht, ob, wie und in welchem Ausmass die Texte vom Absender redigiert und modifiziert wurden, bevor er sie zur Verfügung stellte. So hatte Max uns gegenüber zwar versichert, dass er die SMS vorgängig bereits komplett anonymisiert habe. Es scheint uns jedoch, dass seine Eingriffe in den Text marginal waren und hauptsächlich die Anonymisierung der Arbeitskontakte, nicht aber weitere persönliche Details betrafen. In Bezug auf diesen Aspekt, aber auch in anderen Punkten wirft das Korpus interessante Forschungsfragen auf, auf die hier kurz eingegangen werden soll. Diese betreffen:

(i) die Anonymisierung der Daten;

(ii) die Bedeutung dialogischer Sequenzen für die SMS-Forschung;

(iii) die Funktion, die der SMS-Dialog gegenüber alternativen Kommunikationsformen einnimmt;

\footnotetext{
6 Die Jahreszahl wird vom 'Spender' nicht explizit erwähnt, lässt sich aber aus den Nachrichten selbst
} erschliessen. 
(iv) den potentiellen Beitrag einer qualitativen Studie von SMS-Korpusdaten für die linguistische Forschung.

Was Punkt (i), die Anonymisierung der Daten, betrifft, so konnten wir feststellen, dass die Mehrzahl der Personennamen, einbegriffen die Vornamen der Kinder von Max und Petra aus vergangenen Beziehungen, unverändert in der uns zur Verfügung gestellten Korrespondenz enthalten waren. Dasselbe gilt für etliche Ortsnamen. Alle Anonymisierungen (Namen, Telefon-/Faxnummern, E-Mail-Adressen usw.), die zur Einspeisung der Daten in unser Korpus notwendig waren, haben wir deshalb selbst durchgeführt. Dass dies umso notwendiger ist, als es sich um sensible Daten handelt, zeigt das Subkorpus in anschaulicher Weise. In der Tat führt uns dieser SMS-Dialog mitten ins Privatleben eines Paares am Anfang einer Liebesbeziehung - einer Beziehung, die sich in Bezug auf einen der Korrespondenten als ausserehelich erweist und deshalb eine Reihe von Komplikationen mit sich bringt (etwa was die Wahl der Orte für die Treffen der Partner betrifft). Die SMS enthalten überdies eine Reihe von sehr persönlichen Informationen zu den beteiligten Personen, allen voran zu den Schreibern (berufliche Schwierigkeiten und Karrieresituation, familiäre und gesundheitliche Probleme, finanzielle Angelegenheiten) und deren Angehörigen (z.B. der Kinder). Auch andere persönliche Details (z.B. Mobiltelefonie-Anbieter, Automobil-Marke, ausgeübte Sportarten, Freizeitbeschäftigungen, Reisen o.Ä.) lassen sich über die SMS erschliessen. Eine Bereitstellung des Subkorpus für Forschungszwecke ist aufgrund dieser delikaten Datenlage problematisch. Denn auch wenn man davon ausgehen kann, dass die Daten lediglich einem eher überschaubaren Fachpublikum bekannt werden dürften, ist es aus ethischen Gründen nicht zulässig, das Korpus so zu belassen. Eine radikale Anonymisierung hat aber zur Folge, dass die Korrespondenz nicht mehr in authentischer Form vorliegt. Doch ist der dialogische Charakter dieser Sequenzen (Punkt ii) aus linguistischer Sicht äusserst aufschlussreich, weshalb wir zumindest auf ausgewählte Sequenzen daraus eingehen möchten. Einige Anmerkungen seien daher im Folgenden gemacht:

Auf den ersten Blick zeigte sich bereits, dass die beiden Personen zwei unterschiedliche Schreibstile pflegen: Petra, die knapper, sparsamer schreibt als Max, lässt systematisch die Grussformeln weg, während ihr Partner seine Nachrichten gerne mit folgenden Worten beginnt (v.a. dann, wenn der SMS-Dialog nach einem längeren zeitlichen Unterbruch wieder aufgenommen wird): "Chérie", "Petra, Chérie", "Bonjour, Chérie", "Bonjour mon Amour" oder (in ironischer Weise) "Chère Madame". Von besonderem Interesse sind in den SMSDialogen die Frage-Antwort-Sequenzen, aber auch die Organisation der Sprechakte (Danken, Befehlen usw.) und die Art und Weise der Referenz auf den jeweiligen Gesprächsgegenstand. Ein Beispiel hierfür wird in (2) gegeben:

(2) Je vais $l a$ laisser à $\mathrm{X}$ et je prends un billet jusqu'à $\mathrm{Y}$ et on se retrouve a $\mathrm{Z} \mathrm{c}$ est plus simple je crois. bisous ${ }^{7}$

Die referentielle Bedeutung des Klitikons la ist hier ohne Kenntnis der vorausgehenden Nachricht nicht erschliessbar, sie erklärt sich erst durch den Kontext (es geht um die Frage, wo Petra ihr Auto abstellen kann). Gleiches gilt für die Antwort-SMS von Max: "C'est aussi bien. Prends-le jusqu'à X-aéroport, 1ère classe. [...]", in der das Pronomen le auf das von Petra eingeführte indefinite Nominalsyntagma ("je prends un billet") referiert. Dieses Beispiel führt uns auf anschauliche Weise vor Augen, dass die systematische Analyse anaphorischer Verbindungen nur auf der Grundlage dialogischer SMS-Daten verlässlich vorgenommen werden kann. Ebenso erklären sich elliptische Aussagen oder Konstruktionen mit Null-Objekt nur bei Kenntnis des Kontexts (z.B. die im Korpus häufige Aussage "tu me redis", "tu me rediras" mit Null-Objekt). Die Bedeutung des dialogischen Kontexts zeigt sich auch im

\footnotetext{
${ }^{7}$ Kursivsetzung (hier wie in den folgenden Beispielen) von uns.
} 
folgenden Beispiel, in dem sich das Syntagma D'autant plus auf Informationen aus einer vorausgehenden SMS von Petra bezieht:

(3) D'autant plus dommage qu'il n'a pas moyen de te joindre. [...]

Selbstverständlich ist es voreilig, hieraus abzuleiten, dass jegliche Informationen, die für das Verständnis referentieller Angaben in den SMS-Nachrichten notwendig sind, in den Nachrichten selbst enthalten seien. Oftmals beziehen sich die Schreiber auch auf ein Geschehen, welches ausserhalb der SMS-Kommunikation, in anderen Kommunikationsformen, stattfindet. So ist es auffällig (Punkt iii), wie oft der zwischen den beiden Personen alternierende Gebrauch der Kommunikationsformen (Brief, Fax, E-Mail, Telefongespräch sowie Face-to-Face-Gespräch) in den SMS thematisiert wird. Beispielsweise bedankt sich Petra bei Max für die "superbe lettre", die sie von ihm erhalten hat; Max seinerseits kündigt Petra mittels SMS "un nouveau mail" an. Oftmals dient eine per SMS verschickte Mitteilung auch dazu, den Versand oder Empfang einer auf anderem Weg übermittelten Nachricht zu bestätigen: "je te sms quand je te faxe", "je viens de te faxer mon cv", "non, je vais lire le mail, merci", "je t'envoie un sms quand tu peux me tel demain", "peux-tu m'appeler [d.h. auf telefonischem Weg] je m'endors [...]".

Abschliessend seien noch einige Bemerkungen zu den Möglichkeiten einer qualitativen Auswertung der Korpusdaten angefügt (Punkt iv), die - unter Berücksichtigung der angesprochenen Schwierigkeit hinsichtlich der Anonymisierung der Daten - durchgeführt werden könnte. Weiter oben wurde bereits erwähnt, dass Deutsch die Erstsprache von Max ist, Französisch ist seine Zweitsprache. Wie der folgende Auszug zeigt, beherrscht er die französische Sprache auf durchweg hohem Niveau:

(4) Bonjour Chérie,

Puis-je conclure du fait apparent que tu n'as pas encore trouvé le temps pour me faire un SMS que tu es en hâte pour aller à $\mathrm{X}$ ou que tu dors encore ? [...]

Nur die Form "tu es en hâte" (anstelle von "tu es pressée") verrät in diesem Text die nichtmuttersprachliche Kompetenz des Schreibers. Dagegen kommen in anderen SMS von Max die charakteristischen Probleme eines Deutschsprechers in einer L2-Varietät des Französischen stärker zum Vorschein. Hier einige Beispiele:

(5) Tu me fais heureux (= tu me rends heureux)

(6) Toi aussi et rêves doucement (= fais de doux rêves)

(7) Je commence lentement à avoir pitié avec toi ! (= à avoir pitié de toi)

(8) Où es-tu, je me fais des soucis. (= du souci)

In einigen SMS zeigen sich auch Besonderheiten in der Interpunktion, die darauf schliessen lassen, dass der Schreiber in der Kommasetzung den Regeln der deutschen, nicht der französischen Orthographie folgt (vgl. die Beispiele (9), (10) und (11)). Auch ist die Tempuswahl an mehreren Stellen markiert. So würde man in (12), (13) und (14) korrekterweise das Passé composé anstelle der Imperfektform erwarten (vgl. c'est bien allé; comment c'est allé). Solche und ähnliche L2-spezifische Besonderheiten ${ }^{8}$, die hier nur exemplarisch aufgeführt werden können, illustrieren den Beitrag, den die Analyse von SMSDaten für linguistische Fragen (z.B. zur Analyse von Interferenzphänomenen) leisten kann.

(9) Tu peux déjà estimer, quand tu devras repartir?

\footnotetext{
8 Dazu gehören etwa Fehler beim Gebrauch der Inversion des Subjektklitikons: "dois-je me faire des soucis ou faisait ton portable suisse des problèmes" (anstatt: "ou ton portable suisse faisait-il des problèmes"); interessant ist auch das Beispiel, in dem Max einem initialen que fälschlicherweise, möglicherweise als Hyperkorrektur, eine Inversion folgen lässt: "Ô, qu'ai-je eu de la chance" (anstatt: "que j'ai eu de la chance").
} 
(10) je ne suis pas sûr, ou je te rencontre : [...]

(11) Petra Chérie,

(12) Ça allait bien avec la Jeep louée ?

(13) Comment ça allait cette après-midi ?

(14) Ça allait bien aujourd'hui ?

Abschliessend sei nochmals betont, dass die Auswertung dialogischer Sequenzen in der SMSForschung bisher nur selten Berücksichtigung fand. Die Arbeit mit den Subkorpora, die unserem Projekt zur Verfügung stehen, kann in diesem Bereich eine Lücke schliessen. Da es sich dabei aber, wie dieser kleine Einblick gezeigt hat, um sehr private Daten handelt, können die SMS nur auszugsweise für Forschungszwecke herangezogen werden.

\section{$4 \quad$ Literaturüberblick und Forschungsfragen}

An dieser Stelle soll noch ein Blick auf die mittlerweile umfangreich vorhandene Literatur zur SMS-Forschung geworfen werden. Hier können wir unmittelbar anschliessen an einen Artikel von Thurlow/Poff (im Druck) mit dem Titel "The Language of Text Messaging", in dem ein aktueller Literaturüberblick zur SMS-Forschung gegeben wird. Die Untersuchungen, die in diesem Beitrag vorgestellt werden und bis ins Jahr 2009 reichen, beziehen sich auf die SMSKommunikation in Finnland, Schweden, Norwegen, Dänemark, Frankreich, Griechenland, Deutschland, Italien, Südafrika, Nigeria, Neuseeland, Kuwait, Malaysia, Japan, Korea, China, Taiwan, Hongkong, Grossbritannien und in den USA. Die Schweiz allerdings bleibt unerwähnt. Da die Autoren an anderer Stelle durchaus nicht-englischsprachige Titel berücksichtigen, hängt dies nicht mit der Publikationssprache in den einschlägigen Beiträgen (z.B. Deutsch) zusammen. Vielmehr gibt es bisher nur wenige empirische Untersuchungen, die in der SMS-Forschung auf die spezifische Schweizer Sprachsituation Bezug nehmen. Dabei handelt es sich durchweg um solche Arbeiten, die sich mit der Analyse von Textnachrichten in der deutschsprachigen Schweiz befassen, nicht aber mit der SMSKommunikation in den anderen Sprachregionen der Schweiz. Wegen der - im Vergleich zu den anderen Landesteilen der Schweiz stark ausgeprägten - Diglossie in der Deutschschweiz gelten hier spezifische Bedingungen, die sich in der privaten Alltagskommunikation (und die SMS-Kommunikation ist primär eine solche) niederschlagen. Die SMS-Forschung trägt diesem Umstand mittlerweile in einigen Arbeiten Rechnung (siehe dazu weiter unten).

Was die Verwendung des Italienischen und Französischen in der SMS-Kommunikation betrifft, so liegen bislang keine Untersuchungen vor, in denen ein Bezug zur Schweiz hergestellt würde. Auf das Italienische als Kontaktsprache in der computervermittelten Kommunikation (= CMC) in der Deutschschweiz geht zwar Adrian Stähli (2009) ein, Textnachrichten werden dabei aber nur am Rande berücksichtigt. Doch immerhin gibt es einige Studien zur SMS-Kommunikation in italienischer Sprache, die als Orientierung für die Analyse der italienischsprachigen SMS in unserem SMS-Korpus dienen können. Hier seien exemplarisch zwei Titel genannt: Elena Pistolesi publizierte im Jahr 2004 eine vergleichende Studie zum Thema Il parlar spedito: l'italiano di chat, e-mail e SMS. Von Flavia Ursini (2005) stammt die Publikation "Tra scritto e parlato: i 'messaggi brevi' tra telefoni cellulari". Für das Französische (aber wiederum ohne Bezug zur Schweiz) sind u.a. die hervorragenden Arbeiten von Jacques Anis zu nennen, der typographische Merkmale beschreibt, die auch in den SMS im Schweizer Korpus vorkommen. Dazu gehören Rebusschreibungen vom Typ $2 m 1$ (= demain) und $7 n 8$ (= cette nuit), das Auslassen von Vokalen (vgl. je t'm (= je t'aime)) oder phonetische Schreibungen, wie sie in j'esper ke tu va bien auftreten (vgl. Anis 2007: 97). Weiter seien Arbeiten erwähnt, die auf Daten aus dem belgischen sms4science-Projekt basieren. Darunter fallen die von Cédrick Fairon et al. (2006) vorgelegte Monographie Le 
langage SMS und die Arbeiten von Louise-Amélie Cougnon. In einem Aufsatz von 2010 vergleicht Cougnon - in Ko-Autorenschaft mit Gudrun Ledegen - die Varietät des Französischen im belgischen Korpus mit den SMS aus dem Korpus von La Réunion (vgl. Cougnon/Ledegen 2010), in einem anderen Aufsatz analysiert sie die belgischen Korpusdaten auf syntaktischer und graphisch-phonetischer Ebene (vgl. Cougnon 2008). Erwähnt sei in diesem Zusammenhang auch, dass an der Universität Zürich eine erste Qualifikationsarbeit zum belgischen SMS-Korpus verfasst wurde. Dabei handelt sich um die Lizenziatsarbeit von Theo Zimmermann (2009) mit dem Titel Le langage SMS - une nouvelle varieté écrite de la langue française? Une analyse empirique basée sur un corpus de 30'000 SMS sous considération particulière de la relation phonie-graphie. Angemerkt sei weiter, dass es bislang keine Forschung zur SMS-Kommunikation im Rätoromanischen gibt. Angesichts der Tatsache, dass diese Sprache eine so geringe Zahl von Sprechern hat, verwundert das nicht. ${ }^{9}$ Wir möchten aber darauf aufmerksam machen, dass in unserem Schweizer sms4scienceKorpus 214 SMS vorliegen, in denen das Rätoromanische die Hauptsprache ist. Matthias Grünert, der für das vorliegende Heft einen Beitrag verfasst hat, analysiert diese SMS aus linguistischer Sicht und betritt damit in der SMS-Forschung absolutes Neuland.

Kommen wir nun zurück zu der Literaturübersicht von Crispin Thurlow und Michele Poff, die wir im Folgenden durch einige neuere Titel ergänzen werden. ${ }^{10}$ Ausserdem werden wir einige typische Merkmale von SMS-Texten nennen, auf die in der Forschung immer wieder hingewiesen wird. Genauere Hinweise hierzu finden sich in den im Folgenden referierten Arbeiten (z.B. Tagg 2009, siehe auch Dürscheid/Wagner/Brommer 2010). ${ }^{11}$ Hier beschränken wir uns weitgehend auf einen Literaturüberblick.

Der Beitrag von Thurlow/Poff (im Druck) wurde für das von Susan Herring et al. herausgegebene Handbook of the Pragmatics of Computer Mediated Communication verfasst, das voraussichtlich im Jahr 2012 erscheinen wird. Wie der Titel dieses Handbuchs bereits zeigt, wird die SMS-Kommunikation hier, wie in der anglophonen Literatur generell, der computervermittelten Kommunikation zugerechnet. Das lässt sich damit rechtfertigen, dass auch bei Handy-zu-Handy-Nachrichten die Übermittlung der Daten über den Computer des Mobilfunkbetreibers läuft. Ein anderes Argument ist, dass es sich in beiden Fällen um eine Kommunikation handelt, deren sprachliche Merkmale in einem direkten Zusammenhang mit den medialen Bedingungen stehen, unter denen sie sich vollzieht (vgl. hierzu ausführlich Herring 2007). Thurlow/Poff (im Druck) weisen allerdings zu Recht darauf hin, dass ein Unterschied gemacht werden müsse zwischen dem, was technisch möglich ist, und dem, was der Nutzer mit der Technologie tatsächlich mache. So treten einige Merkmale der SMSKommunikation (z.B. Ellipsen) vor allem dann auf, wenn der Nutzer das Handy dialogisch einsetzt, also z.B. unmittelbar nach Erhalt einer SMS eine Antwort-SMS schreibt. Aber auch wenn das nicht der Fall ist, dürfen die Merkmale nicht isoliert betrachtet werden, sondern müssen in Bezug gesetzt werden zum Kontext, in dem sie auftreten, und zum demographischen Hintergrund der Schreiber. ${ }^{12}$

\footnotetext{
${ }^{9}$ Der letzten Volkszählung zufolge, die aus dem Jahr 2000 datiert, liegt die Wohnbevölkerung schweizerischer Nationalitat mit Hauptsprache Rätoromanisch bei 33'868 Personen (=0.6\%). Quelle: Bundesamt für Statistik. Siehe unter http://www.bfs.admin.ch/bfs/portal/de/index/news/publikationen.html?publicationID=1737 $<22.10 .2011>$.

10 Eine solche neuere Arbeit, die auf der Analyse von 3'000 SMS aus dem Hebräischen beruht und von Thurlow/Poff (im Druck) noch gar nicht berücksichtigt werden konnte, stammt von Borochovsky-BarAba/Kedmi (2010).

${ }^{11}$ Die Arbeit von Caroline Tagg ist unveröffentlicht, kann aber über das Internet heruntergeladen werden (s. Literaturverzeichnis). Sie ist nicht nur korpuslinguistisch gut fundiert, sondern bietet einleitend auch einen hervorragenden Forschungsüberblick.

12 Insofern ist es gerechtfertigt, wenn Thurlow/Poff (im Druck) am Ende ihres Artikels dafür plädieren, dass der Interaktionszusammenhang in der Forschung eine stärkere Berücksichtigung finden muss: "[I]t is also important
} 
Welches sind nun die typischen Merkmale der SMS-Kommunikation? Dazu gehören auf graphischer Ebene die Verwendung von Sonderzeichen (Emoticons, Rebusschreibungen), Abkürzungen, Satzzeichenwiederholungen und abweichenden Laut-Buchstabenzuordnungen, auf stilistischer Ebene der Gebrauch umgangssprachlicher Ausdrücke, Dialektismen, Ellipsen und anderer, konzeptionell mündlicher Ausdrucksmittel. ${ }^{13}$ Viele dieser Merkmale treten auch in der privaten Internetkommunikation auf (vgl. beispielsweise die Arbeit von Beat Siebenhaar (2006) zur Chatkommunikation), es ist also keineswegs so, dass sie nur deshalb vorkommen, weil der Platz in einer SMS beschränkt und die Tastatureingabe am Handy umständlich ist. Ausserdem kommen je nach Datengrundlage Merkmale hinzu, andere fallen weg. So spielt in den Arbeiten zur SMS-Kommunikation in der Deutschschweiz die Tatsache, dass ein Grossteil der SMS in Dialekt verfasst ist, eine wichtige Rolle (vgl. Spycher 2004). Andererseits kann in der SMS-Forschung, die auf englischen Daten basiert, nicht von Inflektivkonstruktionen (vgl. mich ganz doll freu) die Rede sein, weil es diese Konstruktion im Englischen nicht gibt. So werden in der Dissertation von Tagg (2009), in der auf der Basis eines umfangreichen Korpus von 10'626 englischsprachigen SMS zahlreiche Ausdrucksmittel diskutiert werden, die für die Handykommunikation im Englischen charakteristisch sind, Inflektivkonstruktionen nicht erwähnt.

Thurlow/Poff (im Druck) nennen im Abschnitt "Pragmalinguistic Contexts" mit Bezug auf die einschlägige Literatur und auf die Sprache, die jeweils untersucht wird, eine Reihe weiterer interessanter Merkmale der SMS-Kommunikation, die hier nicht alle referiert werden können. Beispielsweise geben sie Hinweise auf Studien, in denen typische Begrüssungs- und Verabschiedungsmuster in SMS untersucht wurden (so für das Italienische in der Arbeit von Spagnolli/Gamberini 2007). Dazu sei ergänzend noch die Dissertation von Carmen Frehner (2008) genannt, die im Literaturüberblick von Thurlow/Poff (im Druck) nicht erwähnt wird. In dieser medienlinguistisch ausgerichteten Arbeit, die SMS, MMS, E-Mails und Telegramme untersucht, werden SMS-typischen Begrüssungs- und Verabschiedungsformeln zwei separate Kapitel gewidmet (vgl. Frehner 2008: 91-93, 125-128).

Weitere für die SMS-Forschung relevante Themen skizzieren Thurlow/Poff (im Druck) unter den Überschriften "Cross-cultural contexts", "Interactional contexts" und "Metalinguistic contexts". Zwar ist diese Abgrenzung nicht immer trennscharf, dennoch vermittelt sie eine gute Übersicht über die zahlreichen Arbeiten in der SMS-Forschung und ordnet sie jeweils einem der drei Diskurszusammenhänge zu. Zum ersten Themenfeld zählen die Autoren soziolinguistisch ausgerichtete Arbeiten, in denen, wie auch im Projekt sms4science, demographische Angaben erhoben und diese vergleichend in die Interpretation der Daten einbezogen werden (so z.B. Untersuchungen zu der Frage, ob es geschlechtsspezifische Unterschiede in der SMS-Kommunikation gibt). ${ }^{14}$ Auch hier sei eine neuere Arbeit genannt, die von den Autoren nicht berücksichtigt werden konnte: Darin untersucht Markus Bieswanger auf der Basis eines Korpus von 646 deutschsprachigen SMS, ob männliche und

that scholars lead the way in resisting a 'superficial fascination' with technology - and, in the case of texting, with fleeting linguistic curiosities - in favour of a deeper engagement with the cultural contexts and communicative practices which give both technology and language their real meaning."

13 Vgl. zur konzeptionellen Mündlichkeit resp. Schriftlichkeit die grundlegenden Begriffsklärungen von Koch/Oesterreicher (1994). Zu einer kritischen Auseinandersetzung mit diesem Modell und zu seiner Anwendung auf die Internet- und Handykommunikation vgl. Dürscheid (2003).

14 Das Korpus, das aus dem Jahr 2001 datiert, stellt nur einen Ausschnitt aus einem weitaus grösseren Korpus von 1'500 SMS dar, das über das Internet frei verfügbar ist. Siehe hierzu unter http://www.mediensprache.net/archiv/corpora/sms_os_h.pdf $<22.10 .2011>$. Dieses Korpus bildet die Grundlage für eine Arbeit, die im Jahr 2001 in der elektronischen Zeitschrift Networx erschien (vgl. Schlobinski et al. 2001). Darin werden viele Merkmale beschrieben (z.B. Smileys, Ellipsen), die auch in den Folgearbeiten immer wieder genannt werden, und es wird schon damals betont, dass einige der Merkmale aus der Chatkommunikation bekannt sind (vgl. Schlobinski et al. 2001: 6). 
weibliche SMS-Schreiber unterschiedliche Präferenzen im Gebrauch von Abkürzungen zeigen (vgl. Bieswanger 2010). Zum zweiten Themenfeld gehören Studien, in denen SMSDialoge untersucht werden (vgl. Androutsopoulos/Schmidt 2002), zum dritten solche Arbeiten, in denen die SMS-Kommunikation auf einer Metaebene betrachtet wird. In diesen Arbeiten wird aus wissenschaftlicher Sicht meist dafür argumentiert, dass das häufige Schreiben von Textnachrichten keinen negativen Einfluss auf den Gebrauch der Standardsprache habe (so z.B. in der entwicklungspsychologischen Studie von Plester/Wood/Puja 2009). Zu diesem Befund kommen auch andere, von Thurlow/Poff nicht berücksichtigte linguistische Arbeiten neueren Datums (z.B. Shafie/Darus/Osman 2010, Dürscheid/Wagner/Brommer 2010).

Alle hier genannten wissenschaftlichen Untersuchungen zur SMS-Kommunikation stammen aus den letzten 10 Jahren. Arbeiten, die zurück bis in die 90er Jahre des letzten Jahrhunderts reichen, gibt es nicht. So umfasst die SMS-Bibliographie, die sich auf der Website des sms4science-Projekts findet, nur die Jahre 1999 bis 2010, und auch in der umfangreichen Bibliographie zur Arbeit von Thurlow/Poff (im Druck) gibt es keine älteren Titel. Das verwundert nicht. Zwar existiert der SMS-Dienst seit den 1990er Jahren, aber erst seit der flächendeckenden Verbreitung von Mobilfunk-Endgeräten und der Senkung der Mobilfunkgebühren wurde die SMS-Kommunikation in grösserem Umfang genutzt. Das spiegelt sich auch in der Forschung. In der germanistischen SMS-Forschung beispielsweise datieren die ersten Arbeiten aus dem Jahr 2001. Hierzu zählt die bereits erwähnte Studie zur SMS-Kommunikation in einer Gruppe von fünf Personen (Androutsopoulos/Schmidt 2002). Diese Untersuchung, die auf der Auswertung von 934 SMS basiert, wurde zwar erst 2002 veröffentlicht, war aber bereits 2001 über das Internet zugänglich.

Damit kommen wir zum letzten Punkt, zur SMS-Forschung in der Schweiz. Wie weiter oben bereits erwähnt, sind uns nur solche Arbeiten bekannt, die auf Daten aus der deutschsprachigen Schweiz Bezug nehmen. Die ersten Publikationen, die hierzu erschienen, stammen von Spycher (2004) und Braun (2006). In Samuel Spychers Untersuchung werden, auf der Basis von 202 SMS, die charakteristischen sprachlichen Merkmale dieser Nachrichten herausgearbeitet. Die SMS stammen von 33 Gymnasiasten im Alter von 17 bis 21 Jahren und sind fast durchgängig in Dialekt verfasst. Auch Bettina Braun beschreibt in ihrer varietätenlinguistischen Studie SMS-typische Merkmale (syntaktische und lexikalische Kurzformen, von der Orthographie abweichende Schreibungen). Sie untersuchte 206 SMS, die ihr von Sekundarschülern, d.h. von 13- bis 15-jährigen Schülern, zur Verfügung gestellt wurden. Beide kommen zu dem Ergebnis, dass der Dialektgebrauch generell sehr hoch ist, er liegt in ihren Korpora jeweils bei über 90\%. Dies hängt unseres Erachtens nicht nur, wie Braun (2006: 101) betont, mit der identitätsstiftenden Funktion des Dialekts zusammen. Ein Grund ist auch der, dass sich die Alltagskommunikation in der Deutschschweiz in Dialekt vollzieht. Und da die SMS-Kommunikation eine solche Alltagskommunikation ist, die häufig genug dazu dient, Verabredungen zu treffen, sich zu erkundigen, wie es dem anderen geht, was er gerade macht oder wo er ist, verwundert es nicht, dass solch private Mitteilungen in Dialekt geschrieben werden.

Weitere Forschungsergebnisse zur Handykommunikation in der Deutschschweiz werden in den empirischen Untersuchungen von Frehner (2008) und Dürscheid/Wagner/Brommer (2010) präsentiert. Die Zürcher Dissertation von Carmen Frehner basiert auf einem Vergleich deutscher, schweizerdeutscher und englischer E-Mails und SMS und diskutiert in Anlehnung an das Modell von Koch/Oesterreicher (1994) Aspekte der konzeptionellen Mündlichkeit in diesen Texten. Weiter findet sich darin eine Analyse von SMS-Texten, die zu komplexen Nachrichten aneinandergefügt wurden (vgl. Frehner 2009: 123-146). Damit kann Carmen Frehner zeigen, dass Schreiber auch dann Zeichen einsparen und verkürzte Schreibweisen verwenden, wenn sie aufgrund der Verknüpfung mehrerer SMS genügend Platz zur 
Verfügung hätten. Inwieweit typische SMS-Phänomene auf Ökonomiebestrebungen zurückzuführen sind und nicht vielmehr andere Ursachen haben (z.B. so zu schreiben wie die anderen), bleibt vor diesem Hintergrund noch $\mathrm{zu}$ diskutieren. Im SNF-Projekt von Dürscheid/Wagner/Brommer (2010) zur Schreibkompetenz von Schweizer Schülern werden SMS-Texte von 14- bis 19-Jährigen untersucht und die darin auftretenden graphischen und stilistischen Merkmale mit anderen Texten aus der privaten Alltagskommunikation verglichen (u.a. E-Mails, Chatbeiträge). Von den 634 SMS, die in das Korpus eingegangen sind, liegen nur acht Texte in Standardsprache vor, alle anderen sind durchgängig in Dialekt verfasst. Interessant ist, dass in diesen SMS abweichende Lautbuchstaben-Zuordnungen auftreten, die keineswegs auf die dialektale Verschriftung zurückgeführt werden können. Dazu gehört die von den Jugendlichen aus der Rap-Szene übernommene <sh>-Schreibung (vgl. geshtr statt geschtr), die $<\mathrm{z}>$-Schreibung, die anstelle von $<\mathrm{s}>$ oder $<\mathrm{ts}>$ (vgl. wie gatz? oder Mer geiz guet) steht, und die $<\mathrm{x}>$-Schreibung anstelle von $<\mathrm{s}>$ (vgl. glungnigx anstelle glungnigs). Daraus ist zu folgern, dass die Schreiber die Graphie bewusst einsetzen, um ihr Szenewissen anzuzeigen.

Damit ist der kurze Literaturüberblick abgeschlossen, nun seien noch einige der Forschungsfragen benannt, die auf der Basis des Schweizer sms4science Korpus in den nächsten Jahren (z.B. im Rahmen von Dissertationsprojekten) bearbeitet werden sollen. Zum einen wird das Code-Switching im Zentrum weiterer Untersuchungen stehen. Tritt dieses Phänomen in den Schweizer SMS (im Gegensatz zum belgischen SMS-Korpus z.B.) verstärkt auf? Und wenn ja, worin zeigt es sich und wie ist dies zu erklären? Denn allein aufgrund der Tatsache, dass die Schweiz ein viersprachiges Land ist, kann ja noch nicht angenommen werden, dass auch seine Bewohner im SMS-Schreiben zu einer Mehrsprachigkeit tendieren (s. dazu den Beitrag von Simona Pekarek Doehler in diesem Heft). Eine weitere Forschungsfrage, die in diesen Bereich fällt und in einem Dissertationsprojekt bearbeitet werden soll, bezieht sich auf die Korrelation zwischen dem Dialektgebrauch in den SMS und dem Alter der Schreiber. Verfassen nur jüngere Schreiber ihre SMS in Dialekt? Alle weiter oben genannten Deutschschweizer Studien haben das Schreiben Jugendlicher im Blick, wie sieht es aber mit den SMS aus, die von Angehörigen der mittleren oder älteren Generation verfasst werden? Auch wird unser Korpus die Untersuchung von Sprachkontakt, Sprachbewusstsein und Sprachwandel in sog. 'kleinen' Sprachen (in der Schweiz also im Rätoromanischen und Italienischen) erlauben. Des Weiteren stellt sich die bisher in der Forschung nur am Rande thematisierte Frage nach den grammatischen Eigenschaften der SMS - hier mit Blick auf die grossen Sprachen im Korpus, d.h. auf das Deutsche und das Französische. Unsere Hypothese ist, dass es grammatische Strukturen gibt, die in allen Fällen beibehalten werden (z.B. die Markierung von Subjekt-Verb-Kongruenz), und andere, in denen grammatische Variation unterschiedlicher Art und Motivation anzutreffen ist (z.B. die Auslassung von Artikeln und Präpositionen, die Markierung der Negation und die Struktur von Fragekonstruktionen im Französischen). Wenn diese Hypothese stimmt, dann zeigen sich in den SMS-Texten die einer Sprache eigenen, grammatischen Strukturen, die auch dann weiter präsent sind, wenn das Schreiben in anderer Hinsicht nicht normkonform ist (vgl. dazu auch den Beitrag von Elisabeth Stark in diesem Heft). Es sind dies die von jeglicher Pragmatik unabhängigen Prinzipien, die in der Generativen Grammatik im Rahmen einer autonomen Syntax beschrieben werden können.

\section{5 Überblick über die Beiträge des vorliegenden Heftes}

Im Folgenden soll nun abschliessend noch eine kurze Übersicht über die im vorliegenden Heft versammelten Beiträge präsentiert werden. Einen guten Überblick über das Projekt sms4science in seiner internationalen Dimension bieten Louise-Amélie Cougnon und Thomas François ("Étudier l'écrit sms. Un objectif du projet sms4science"). Die Autoren machen 
einige grundsätzliche Bemerkungen zur Erhebung und zur Aufbereitung der SMS-Daten und zur Zusammenstellung der nationalen Korpora, die Teile eines internationalen Gesamtkorpus von 116.000 authentischen SMS-Nachrichten sind und nun zur Analyse vorliegen. Das Korpus erlaubt es, erstmals systematisch und auf Grundlage grosser, mehrsprachiger Datenmengen (was bisher ein Desiderat in der Forschung war) einige zentrale Fragen zu beleuchten, die den Sprachgebrauch in der interpersonellen Kommunikation betreffen. Wie Cougnon und François aufzeigen, bietet das Korpus verschiedenen Disziplinen der Sprachforschung - exemplarisch werden u.a. die Soziolinguistik und die automatische Sprachverarbeitung genannt - interessante Analysemöglichkeiten. Auch methodologische Aspekte werden im Beitrag angesprochen und anhand einiger Überlegungen zur vergleichenden statistischen Auswertung der Daten vorgestellt (Länge der SMS, Abkürzungspraxen in den beteiligten Sprachregionen).

Elisabeth Stark nimmt in ihrem Artikel "La morphosyntaxe dans les SMS suisses francophones: Le marquage de l'accord sujet - verbe conjugé", dem ersten Beitrag zum Schweizer SMS-Korpus im vorliegenden Heft, eine manuelle Analyse von 400 SMS in französischer Sprache vor und untersucht diese im Hinblick auf ihre Morphosyntax. Starks Interesse gilt v.a. der Markierung von Subjekt-Verb-Kongruenz und der Frage, ob die in Kurznachrichten verwendeten Schreibstrategien möglicherweise einen Einfluss auf die Intaktheit grammatischer Strukturen haben. Morphologische und morphosyntaktische Aspekte der SMS-Kommunikation wurden bisher von der Forschung gar nicht oder nur am Rande berücksichtigt, weshalb der Beitrag auf diesem Gebiet eine Pilotstudie darstellt. Auf der Grundlage des französischsprachigen Datenmaterials nimmt die Autorin eine Analyse verschiedener Typen von Kongruenz vor, aus der sich ergibt, dass in den SMS eine stabile und intakte grammatische Markierung vorliegt, welche sich nur unwesentlich von der Schreibung anderer standardnäherer Texte unterscheidet.

Simona Pekarek Doehler präsentiert im Beitrag "Hallo! Voulez vous luncher avec moi hüt? Le 'code switching' dans la communication par SMS" eine Analyse multilingualer Aspekte der SMS-Kommunikation. Sie konzentriert sich auf Formen und Funktionen von CodeSwitching-Phänomenen im SMS-Korpus (vgl. dazu auch den Beitrag von Moretti und Stähli in diesem Heft). Dabei geht sie von der Fragestellung aus, ob plurilinguales Schreiben in der SMS-Kommunikation spezifische Muster erkennen lässt und sich Code-Switching in Kurznachrichten nur bei in einem engeren Sinn bilingualen Individuen findet oder möglicherweise ein Phänomen darstellt, welches auch bei Schreibern anzutreffen ist, die nicht als zweisprachig definiert würden. Nach einer kurzen Einordnung des Phänomens und einer Bestandsaufnahme seiner in der einschlägigen Literatur beschriebenen sozio-interaktiven Funktionen präsentiert die Autorin eine Analyse der 345 SMS-Nachrichten mit Sprachwechsel aus dem französischen Subkorpus (d.h. SMS mit Französisch als Ausgangssprache). Dabei stellt sie fest, dass das Code-Switching hauptsächlich einzelne Wörter oder feststehende Redewendungen betrifft (etwa Grussformeln), die aufgrund ihrer hohen Geläufigkeit auch von Personen gebraucht werden, die in der alltäglichen Face-toFace-Interaktion in der Regel nicht von mehrsprachlichen Sprachpraktiken Gebrauch machen.

Diese Tendenz wird von Bruno Moretti und Adrian Stähli für das italienische Subkorpus bestätigt, in dem anderssprachige Textteile in ähnlicher Weise vielfach idiomatischen Charakter haben und nicht zwingend eine hohe mehrsprachige Kompetenz der Schreiber voraussetzen. In ihrem Beitrag "L'italiano in contatto con il dialetto e altre lingue. Nuovi mezzi di comunicazione e nuove diglossie" stellen die Autoren einleitend einige der Schreibstrategien in italienischen SMS vor (insbesondere sprachliche Verknappungen mittels Abkürzungen, Akronymen u. Ä.), welche als stilisierende Mittel dieser Kommunikationsform äusserst gängig sind und besonders unter Jugendlichen als 'Identitätsmarker' (vgl. Braun 2006) gelten können. Diese Verknappungsstrategien können in den italienischen 
Kurznachrichten teilweise zu einer Dialektalisierung des Schreibstils führen. Wie die Autoren bemerken, begünstigt die Kommunikationsform SMS das Aufkommen dieser 'neuen' Dialektalität, wobei sich der innovative Gebrauch des Dialekts in vielerlei Hinsicht von einer traditionellen Dialektverwendung unterscheidet und somit auf eine mögliche Restrukturierung des Sprachrepertoires hinweist.

Code-Switching-Phänomene zeichnen erwartungsgemäss auch die rätoromanischen SMS im Korpus aus. Matthias Grünert geht in dem Artikel "Varietäten und Sprachkontakt in rätoromanischen SMS" denn auch auf sprachkontaktbedingte Erscheinungen ein, die typischerweise die gesprochene Sprache charakterisieren und im geschriebenen Rätoromanischen meistens vermieden werden. Nach einer Vorstellung der zum rätoromanischen Subkorpus gehörenden soziodemographischen Daten nimmt der Autor eine differenzierte Analyse des Gebrauchs der regionalen (Haupt-)Varietäten in den 214 rätoromanischen SMS vor. Dies ist im Hinblick auf das Fehlen einer allgemein akzeptierten standardisierten Form der Minderheitensprache Rätoromanisch wichtig. So findet die seit 1982 entwickelte Schriftsprache Rumantsch Grischun nur in 22 Kurznachrichten Verwendung. In den anderen Fällen verwenden die Schreiber die jeweilige regionale Schriftsprache und geben in deren graphischem Code Züge der regionalen Mündlichkeit, teils auch ihres Dialekts wieder. Dieser Aspekt einer schriftlichen Realisierung von Mündlichkeit erweist sich bekanntermassen als allgemein typisch für die SMS-Kommunikation (vgl. Abschn. 4 dieser Einleitung), ist aber hinsichtlich der 'Zersplitterung' der rätoromanischen Regionalvarietäten von besonderem Interesse. Angesichts dieser starken Diversifizierung drängt sich, trotz der geringen Datenmenge, eine Unterteilung in regionale Subkorpora auf, zumal auch Sprachkontaktphänomene, wie der Autor zeigen kann, regional durchaus unterschiedlicher Ausprägung sind.

Die Situation des Rätoromanischen, der kleinsten Sprache in der viersprachigen Schweiz, mag in einem gewissen Sinn, auf einer Mikroebene, als exemplarisch für die sprachliche Vielfalt in der Schweizer SMS-Kommunikation gelten. Die im vorliegenden Heft vorgestellten Untersuchungen widmen sich einigen wichtigen Fragestellungen, die im Zusammenhang mit dieser Kommunikationsform im Schweizer Kontext von zentraler Bedeutung sind. Gleichzeitig zeigen sie auf der Makroebene das Potential und die Wichtigkeit weiterer SMS-Studien an. Diese können, so hoffen wir, zu einem vertieften Verständnis der Phänomene rund um die schriftliche Handy-Kommunikation beitragen.

\section{Literatur}

Androutsopoulos, Jannis/Schmidt, Gurly (2002): "SMS-Kommunikation: Ethnografische Gattungsanalyse am Beispiel einer Kleingruppe". Zeitschrift für Angewandte Linguistik 36: 49-80.

Anis, Jacques (2007): "Neography - Unconventional Spelling in French SMS Text Messages". In: Brenda, Danet/Herring, Susan C. (eds.): The Multilingual Internet Language, Culture and Communication Online. New York, Oxford University Press: 87115.

Bieswanger, Markus (2010): "Gendered language use in computer-mediated communication: Typography in textmessaging". In: Bieswanger, Markus/Motschenbacher, Heiko/Mühleisen, Susanne (eds.): Language in its Socio-Cultural Context. New Exploration in Gendered, Global and Media Uses. Frankfurt a.M., Peter Lang: 157-172.

Borochovsky-Bar-Aba, Esther/Kedmi, Yafit (2010): "The nature of SMS discourse: The case of Hebrew". Folia Linguistica 44/1: 1-30. 
Braun, Bettina (2006): "Jugendliche Identitäten in SMS-Texten". In: Dürscheid, Christa/ Spitzmüller, Jürgen (eds.): Zwischentöne. Zur Sprache der Jugend in der Deutschschweiz. Zürich, Verlag Neue Zürcher Zeitung: 101-114.

Cougnon, Louise-Amélie (2008): "Le français de Belgique dans 'l'écrit spontané'. Approche d'un corpus de 30.000 SMS". In: Travaux du Cercle Belge de Linguistique. http://uclouvain.academia.edu/LouiseAmelieCougnon/Papers/175506/Le-français-de-

Belgique-dans--1-écrit-spontané---Approche-d-un-corpus-de-30-000-SMS <20/10/2011>.

Cougnon, Louise-Amélie/Ledegen, Gudrun (2010): "Une étude comparatiste des variétés du français dans l'écrit SMS (Réunion-Belgique)". In: Abecassis, Michaë1/Ledegen, Gudrun (eds): Les voix des Français: usages et représentations. Colloque AFLS à Oxford en avril 2008. Cambridge: Scholars Publishing: 39-57.

Dürscheid, Christa (2003): "Medienkommunikation im Kontinuum von Mündlichkeit und Schriftlichkeit. Theoretische und empirische Probleme". In: Zeitschrift für Angewandte Linguistik 38: 37-56.

Dürscheid, Christa/Wagner, Franc/Brommer, Sarah (2010): Wie Jugendliche schreiben. Schreibkompetenz und neue Medien. Mit einem Beitrag von Saskia Waibel. Berlin/New York: de Gruyter.

Dürscheid, Christa/Stark, Elisabeth (2011): "sms4science: An international corpus-based texting project and the specific challenges for multilingual Switzerland". In: Thurlow, Crispin/Mroczek, Kristine (eds.): Digital Discourse. Language in the New Media. Oxford, Oxford University Press: 299-320.

Fairon, Cédrick/Klein, Jean René/Paumier, Sébastien (2006): Le langage SMS. Etude d'un corpus informatisé à partir de l'enquête 'Faites don de vos SMS à la science'. Louvain-laNeuve: Presses universitaires de Louvain.

Frehner, Carmen (2008): Email - SMS - MMS. The Linguistic Creativity of Asynchronous Discourse in the New Media Age. Bern: Peter Lang.

Herring, Susan C. (2007): "A Faceted Classification Scheme for Computer-Mediated Discourse".Language@Internet 4. http://www.languageatinternet.org/articles/2007/761 $<17 / 11 / 2010>$.

Koch, Peter/Oesterreicher, Wulf (1994): "Schriftlichkeit und Sprache". In: Günther, Hartmut/Ludwig, Otto (eds.): Schrift und Schriftlichkeit / Writing and its Use. Ein interdisziplinäres Handbuch internationaler Forschung / An Interdisciplinary Handbook of International Research. Berlin/New York: Mouton de Gruyter: 587-604.

Pistolesi, Elena (2004): Il parlar spedito: l'italiano di chat, e-mail e SMS. Padova: Esedra.

Plester, Beverly/Wood, Clare/Joshi, Puja (2009): "Exploring the relationship between children's knowledge of text message abbreviations and school literacy outcome". British Journal of Developmental Psychology 27/1: 145-161.

Schlobinski, Peter et al. (2001): Simsen. Eine Pilotstudie zu sprachlichen und kommunikativen Aspekten in der SMS-Kommunikation (=Networx 22).

http://www.mediensprache.net/de/handysprache/networx/docs/index.asp?id=22 $<23 / 10 / 2011>$.

Shafie, Latisha Asmaak/Darus Norizul Azida/Osman Nazira (2010): "SMS Language and College Writing: The languages of the College Texters". International Journal of Emerging Technologies in Learning 5/1: 26-31.

Siebenhaar, Beat (2006): "Code choice and code-switching in Swiss-German Internet Relay Chat rooms". Journal of Sociolinguistics 10/4: 481-506.

Spagnolli, Anna/Gamberini, Luciano (2007): "Interacting via SMS. Practices of social closeness and reciprocation". British Journal of Social Psychology 46: 343-364.

Spycher, Samuel (2004): 'I schibdr de no...' - Schweizerdeutsche Umgangsformen in der SMS-Kommunikation (=Networx 36). http://www.mediensprache.net/de/ networx/docs/networx-36.asp <20/10/2011>. 
Stähli, Adrian (2009): "Loss, hai rottiert i coglioncini: Mistilinguismo e commutazione di codice nella comunicazione mediata dal computer (CMC) di giovani immigrati italiani di seconda generazione nella Svizzera tedesca". In: Moretti, Bruno/Pandolfi, Elena Maria/Casoni, Matteo (eds.): Linguisti in contatto. Ricerche di linguistica italiana in Svizzera. Atti del convegno. Bellinzona, 16-17 novembre 2007. Bellinzona, Osservatorio linguistico della Svizzera italiana: 183-198.

Tagg, Caroline (2009): A Corpus Linguistics Study of SMS Text Messaging. PhD (unveröff.). Department of English, University of Birmingham. http://etheses.bham.ac.uk/253/ 1/Tagg09PhD.pdf <20/10/2011>.

Thurlow, Crispin/Poff, Michele (im Druck). "The Language of Text Messaging". In: Herring, Susan C./Stein, Dieter/Virtanen, Tuija (Hrsg.): Handbook of the Pragmatics of CMC. Berlin New York: de Gruyter (im Druck). http://faculty.washington.edu/thurlow/ papers/thurlow\&poff\%282010\%29.pdf <20/10/2011>.

Ursini, Flavia (2005): "Tra scritto e parlato: i 'messaggi brevi' tra telefoni cellulari". In: Burr, Elisabeth (ed.): Tradizione \& Innovazione. La linguistica e filologia italiana alle soglie di un nuovo millennio. Atti del VI Convegno Internazionale SILFI (Duisburg 23.6 - 2.07 2000). Florenz, Franco Cesati: 443-455.

Zimmermann, Theo (2009): Le 'langage SMS' - une nouvelle varieté écrite de la langue française? Une analyse empirique basée sur un corpus de 30'000 SMS sous considération particulière de la relation phonie-graphie. Lizenziatsarbeit Universität Zürich (unveröffentlicht). 